\section{On Uniformity of Film Thickness on Rotating Substrates}

\section{B. S. Ramprasad, T. S. Radha, and M. Ramakrishna Rao}

Central Instruments and Services Laboratory, Indian Institute of Science, Bangalore 12, India

For most applications of thin films, uniformity of film thickness over the substrate area is a necessary requirement. An investigation is being carried out in the laboratory to obtain uniform films on optical components. During the course of investigation, it was observed that reported values of geometrical parameters assumed for minimum variation in film thickness, based on the equation derived by Holland and Steckelmacher,' differ widely. Film thickness distribution curves presented by R4acleod 2 are found to be in error. The corrected distribution curves are presented. Also the thickness distribution curves are presented in a form in which suitable geometrical parameters can be chosen readily to yield the desired uniformity in thickness over the area of the substrate.

For a small area surface source the distribution of film thickness on a planar substrate is given by

$$
\begin{aligned}
& t=-. . \quad h \quad h 2(h 2+p 2+R 2) \\
& \sim p \overline{\left.\left[(h 2+p 2+R 2)^{\prime}-4 p 2 R 2\right]\right)^{\prime}}
\end{aligned}
$$

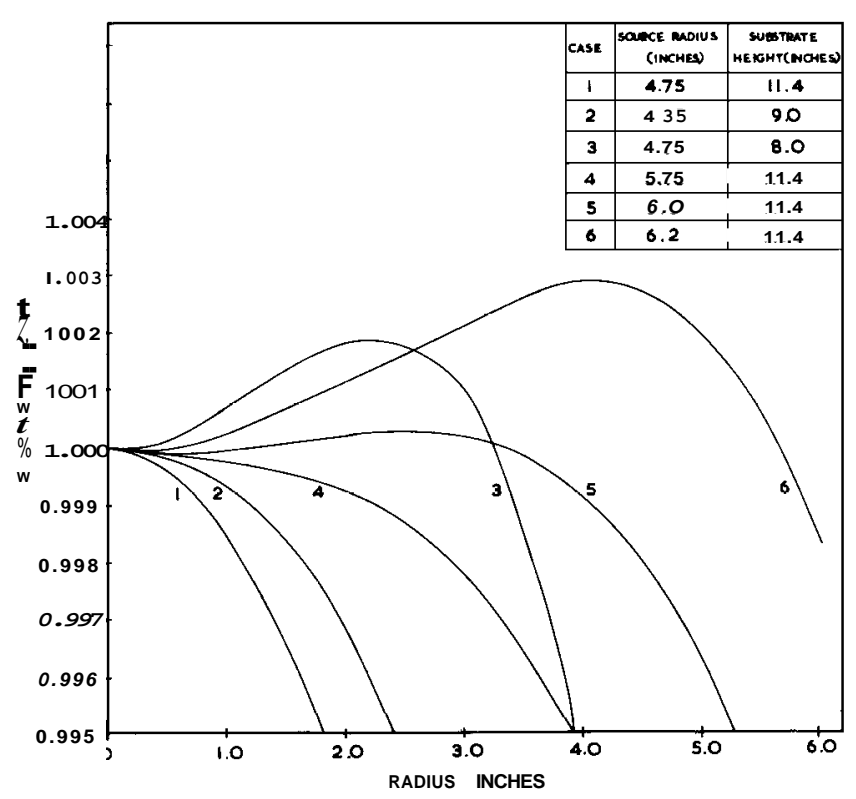

FIGURE 1. Relative thickness vs substrate radius (Macleod2).

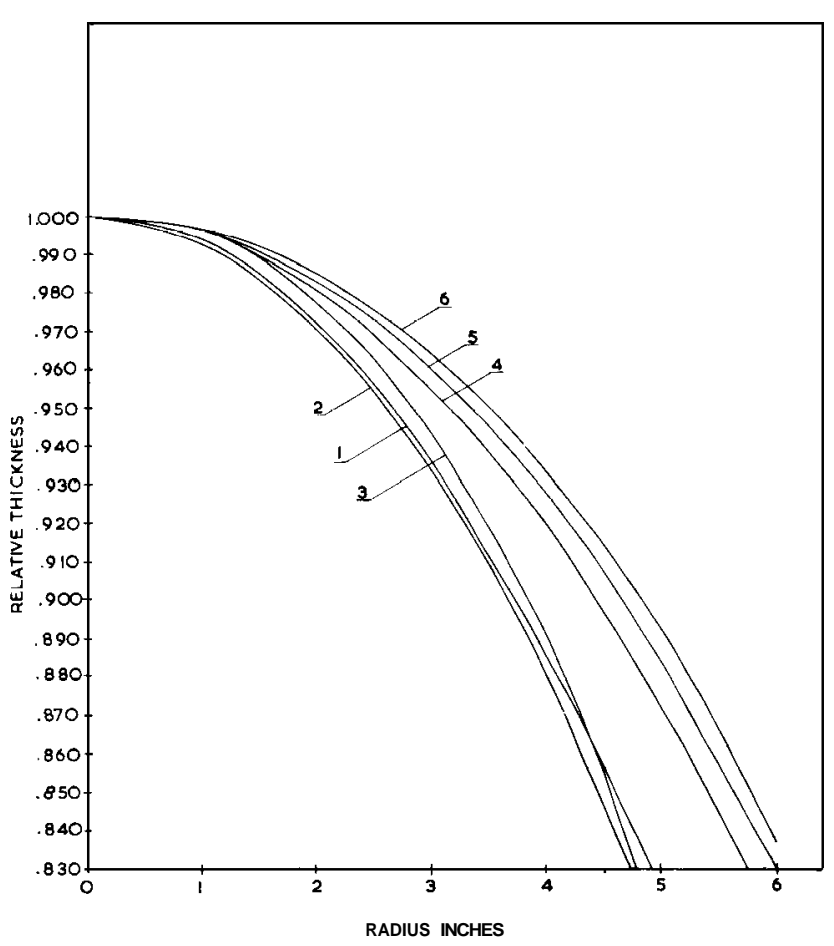

Figure 2. Corrected relative thickness vs substrate radius computed for the values given by Macleod.

where $m=$ mass of evaporant, $\boldsymbol{p}=$ density of evaporant material, $\boldsymbol{h}=$ source to substrate distance, $\boldsymbol{R}=$ distance between source and the axis of rotation of substrate, and $\mathrm{p}=$ distance of the point under consideration from the axis of rotation.

This equation is given in several forms by different workers and they are plotted against different parameters. The utility of these curves lies in choosing the geometrical parameters giving required uniformity of film thickness.

The values in the curves of Macleod2 reproduced in Fig. 1 do not agree with any of the published values. ${ }^{3-7}$ Hence, in order to check, the data for source radius and substrate height given in the insert in Fig. 1 was fed into the computer and the corrected results obtained are plotted in Fig. 2. Table I gives a comparison of some values. It is observed that the values of corrected relative thickness in Fig. 2 are greater than Macleod's reported values.

If we choose case 2 in Table I for $h / R$ ratio of 1.9 the variation in thickness over a radius of $4 \mathrm{in}$. is $0.07 \%$, whereas the corrected value is $7.3 \%$.

A study on uniformity of film thickness is being carried out in the authors' laboratory to find out the optimum geometry for a variation less than $0.5 \%$ over 


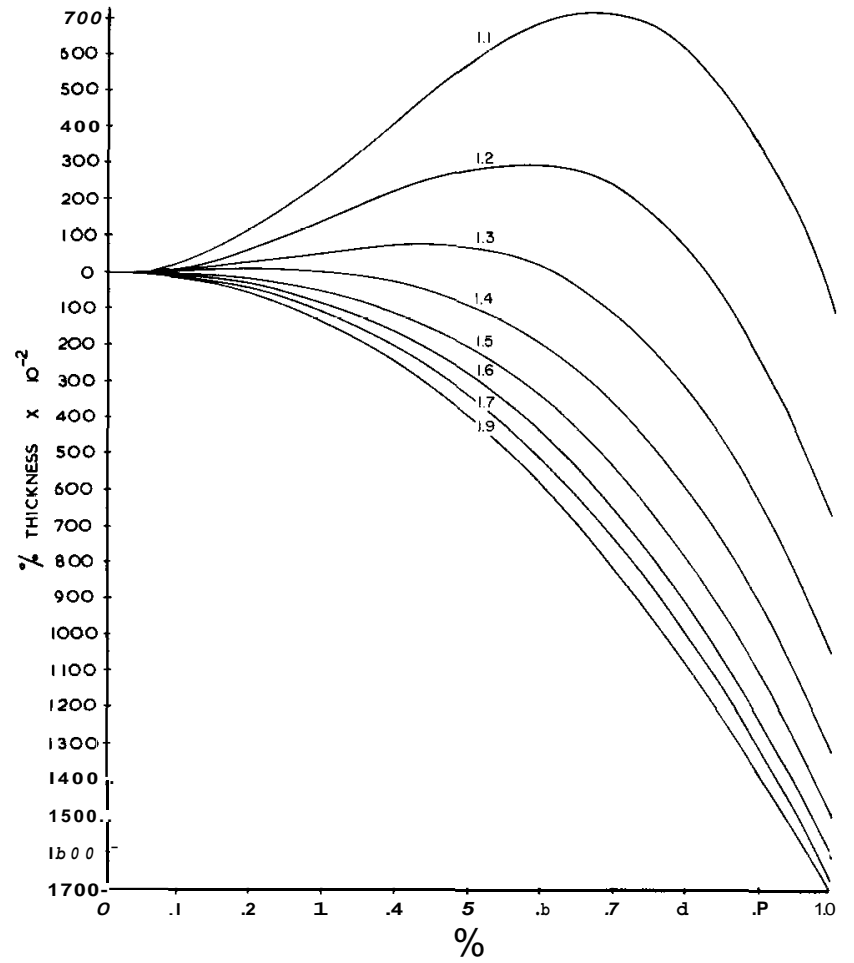

FIGURE 3. Normalized distribution curve giving percentage variation in thickncss.

a substrate area of $4 \mathrm{in}$. diam. During the course of investigation, it was felt that the curves representing variation in thickness $[(t / t,-1) \%]$ against $p / R$ for different ratios of $h / R$ would lead to a choice of experimental parameters to suit the specific requirements. Figure 3 gives the normalized distribution curves for the equation for values of $h / R$ ranging from 1.1 to 1.9 and $p / R$ from 0 to 1.0 .

$$
\frac{t}{t o}=\frac{(h 2 / R 2+1) 2(h 2 / R 2+p 2 / R 2+1)}{[(\boldsymbol{h} 2 / \boldsymbol{R} 2+\boldsymbol{p} 2 / \boldsymbol{R} 2+\boldsymbol{1}) 2-\boldsymbol{4 p} 2 / \boldsymbol{R} 2] \boldsymbol{t}^{\prime}}
$$

The variation of the relative thickness progressively decreases with increasing values of $h / R$. It can be seen in Fig. 3 that minimum variation of thickness occurs around $h / R=1.4$ for a range of $p / R$ values from 0 to 0.5 .
TABle I. Comparison of values of film thickness distribution curve parameters.

\begin{tabular}{ccccccc}
\hline \hline Case & $h / R$ & $h$ & $R$ & $\boldsymbol{p}$ & Macleod2 & Corrected \\
\hline 1 & 1.84 & 11.4 & 6.2 & 4.0 & 1.0030 & 0.9334 \\
2 & 1.90 & 11.4 & 6.0 & 4.0 & 0.9993 & 0.9264 \\
3 & 2.40 & 11.4 & 4.75 & 1.8 & 0.9950 & 0.9761 \\
\hline \hline
\end{tabular}

Further, these curves would facilitate the proper selection of $h$ and $R$ for the desired uniformity, independent of the physical dimensions of the vacuum chamber. For example, consider an application where $0.5 \%$ variation in film thickness from the center to the periphery of given substrate is tolerable. From Fig. 3 any two values of $p / R$ can be chosen for optimum $h / R=1.4$ for $1 \%$ variation from end to end. Since $R$ is a constant for a vacuum chamber the values of $p 1$ and $p z$ can be calculated and their difference gives the diam of the substrate with its center at $+(p l+p z)$. The annular area between $p 1$ and $p 2$ determines the number of substrates which can be accommodated. Four 1.5 in. diam. mirrors can be coated in a 12 in. vacuum chamber with less than $0.5 \%$ variation in film thickness from center to edge, with $R=3.5 \mathrm{in}$. and $h=4.9 \mathrm{in}$. with the centers of the mirrors placed at 1.25 in. from center of rotation. This has been verified experimentally. ${ }^{5}$

\section{Acknowledgment}

The authors are grateful to Dr. S. Dhawan, Director, Indian Institute of Science, for his kind encouragement and keen interest.

\section{References}

${ }^{1}$ L. Holland and W. Steckelmacher. Vacuum, 11. 354 (1952).

2 H. A. Macleod, Thin Film Opticbl Filters (Adam Hilger Ltd., London, 1969), pp. 226, Fig. 9.4.

${ }^{3}$ L. I. Maissel and R. Glang, Handbook of Thin Film Technology (McGraw-Hill, New York, 1970), pp. 55-59.

4 K. H. Behrndt, Transactions of the Tenth National Vacuum Symposium (Macmillan, New York, 1963), pp. 379.

6 B. S.Ramprasad, T. S. Radha, and M. Ramakrishna Rao, Indian J. Technol., 9, 8 (1971).

6 P. H. Lissberger and J. Ring, Opt. Acta, 2, 42 (1955).

7 T. S. Radha, B. S. Ramprasad, and M. Ramakrishna Rao, First National Svmposium on Solid Thin Films, April 1971, Indian Institute of Science, Bangalore. 